\title{
Miliary brain metastases from lung adenocarcinoma as non- enhancing lesions on MRI: a case report and literature review
}

\author{
Zhenchao Huang $^{1 \#}$, Dan Xie ${ }^{2 \#}$, Ping Yang ${ }^{3}$, En'peng Song ${ }^{1}$, Jinhua Zhang ${ }^{1}$, Jianning Chen ${ }^{4}$, Feng Qin ${ }^{1}$ \\ ${ }^{1}$ Department of Neurosurgery, the Third Affiliated Hospital of Sun Yat-sen University, Guangzhou, China; ${ }^{2}$ Intensive Care Unit, the Third Affiliated \\ Hospital of Sun Yat-sen University, Guangzhou, China; ${ }^{3}$ Department of Pathology, Sun Yat-sen University Cancer Center, Guangzhou, China; \\ ${ }^{4}$ Department of Pathology, The Third Affiliated Hospital of Sun Yat-sen University, Guangzhou, China \\ \#These authors contributed equally to this work. \\ Correspondence to: Feng Qin. Department of Neurosurgery, The Third Affiliated Hospital of Sun Yat-sen University, No. 2693, Kaichuang Avenue, \\ Guangzhou 510530, China. Email: brainqin@163.com.
}

\begin{abstract}
Miliary dissemination is common in tuberculosis, but is an extremely rare form of brain metastasis. It is mainly found in patients with primary lung cancer (small cell and adenocarcinoma). Here, we presented a case of miliary metastases of lung adenocarcinoma to the brain without lesion enhancement on MRI after administration of contrast. A 38-year-old Chinese male was diagnosed with lung adenocarcinoma and received chemotherapy monthly for 6 months. At one month after completion of chemotherapy, the patient presented with headache, dizziness, and vomiting. Brain MRI revealed numerous, disseminated, tiny, rounded cystic high-signal intensity lesions on T2-weighted images, and low-signal intensity lesions on T1-weighted images, with no enhancement. In addition, a high signal on T2-weighted images and uneven enhancement with contrast in the hypophysis were noted. A right frontal lobe biopsy revealed miliary metastases originating from primary lung adenocarcinoma, which was consistent with the pathological finding of a bronchial biopsy. However, the patient and his family requested supportive treatments only, and he died 3 months after the diagnosis. In summary, this case indicates that when imaging findings are not consistent with the most likely cause of miliary brain metastasis, a biopsy is necessary to make a definitive diagnosis.
\end{abstract}

Keywords: Miliary brain metastases; MRI; pathological biopsy; lung adenocarcinoma; case report

Submitted Apr 21, 2020. Accepted for publication Nov 16, 2020.

doi: $10.21037 /$ tcr-20-1898

View this article at: http://dx.doi.org/10.21037/tcr-20-1898

\section{Introduction}

Miliary metastases are an unusual form of cerebral metastatic dissemination, and have been very rarely described in the literature. Miliary brain metastases were initially described by Madow and Alpers in 1951 using the term "carcinomatous encephalitis" (1). Since the introduction of MRI, a number of cases have been reported (2-11). In most cases, the brain lesions were obviously apparent on MRI, and enhanced after intravenous administration of contrast medium. Herein, we presented a case of miliary metastases of lung adenocarcinoma to the brain in which the lesions were visible on MRI, but did not enhance, except for those in the hypophysis. We present the following article in accordance with the CARE reporting checklist (available at http://dx.doi. org/10.21037/tcr-20-1898).

\section{Case presentation}

A 38-year-old Chinese male was diagnosed with lung adenocarcinoma in December 2012 by bronchoscopic needle biopsy performed at Sun Yat-sen University Cancer Center. From March to August 2013, he received chemotherapy (pemetrexed $800 \mathrm{mg}$ and carboplatin $360 \mathrm{mg}$ ) monthly for 6 months. In September 2013 he presented with headache, dizziness, and vomiting. MRI showed numerous, disseminated, tiny, rounded cystic 

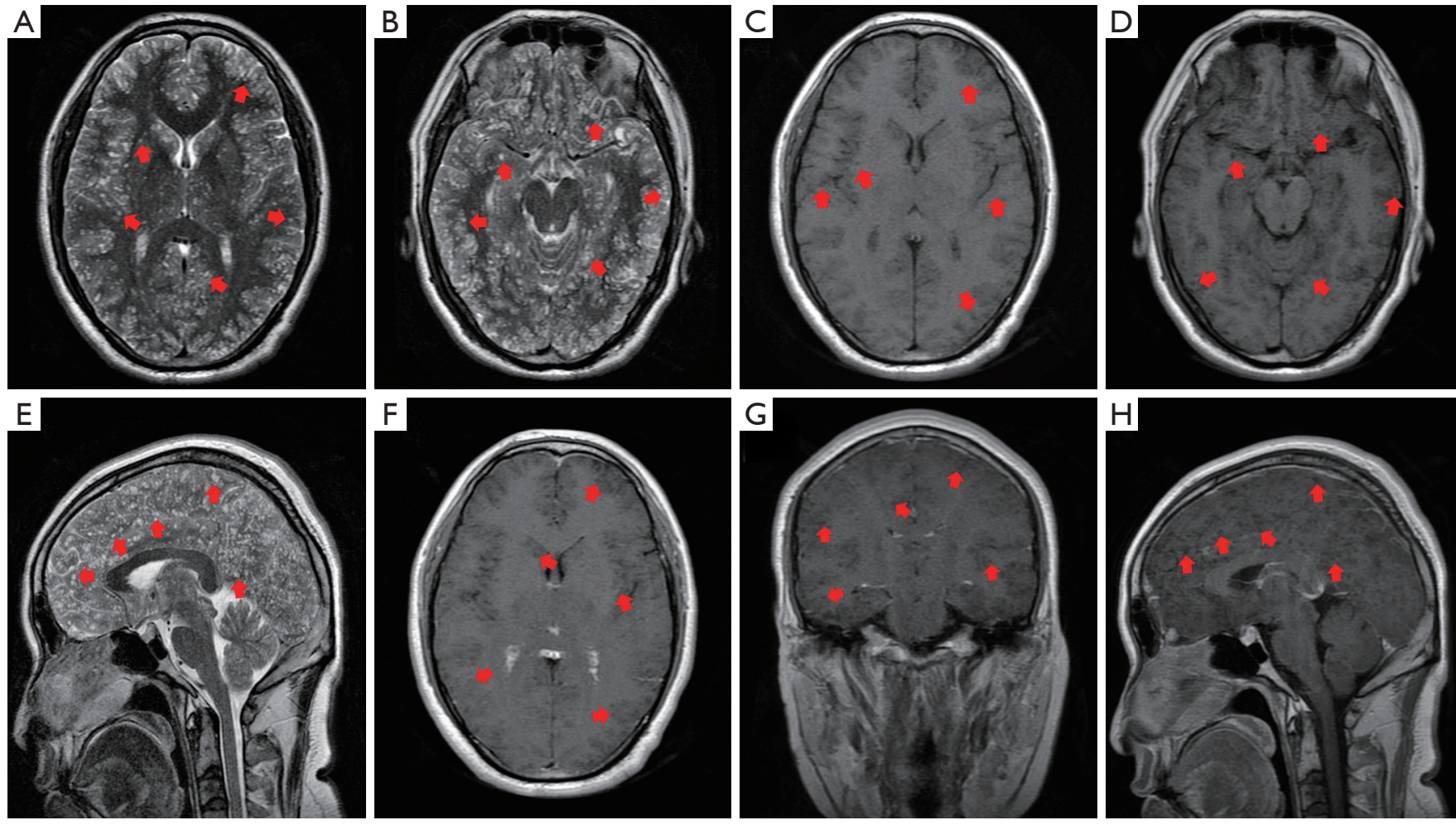

Figure 1 Brain MRI. (A,B) T2-weighted images. (C,D) T1-weighted images. (E) T2-weighted sagittal image. (F,G,H) T1-weighted images after contrast administration. Arrows indicated the lesions.

lesions throughout the brain. Central nervous system (CNS) parasitoses, tuberculosis (TB), and brain metastases were considered in the differential diagnosis. For further diagnosis and treatment, he was transferred to our hospital. This study was approved by the institutional review board (IRB) of our hospital \{approval no. [2020]-02-164-01\}. All procedures performed in studies involving human participants were in accordance with the ethical standards of the institutional and/or national research committee(s) and with the Helsinki Declaration (as revised in 2013). Written informed consent was obtained from the patient.

A repeat lumbar puncture with cerebrospinal fluid (CSF) analysis showed a normal white blood cell (WBC) count, and normal protein, glucose, and chlorine levels, but revealed a high opening pressure $\left(>330 \mathrm{~mm} \mathrm{H}_{2} \mathrm{O}\right)$. Three cytological examinations of CSF specimens obtained at 1 week intervals showed a small number of mature lymphocytes, and no malignant cells. Blood and serological examinations were normal, except for a high carcinoembryonic antigen level $(>100 \mu \mathrm{g} / \mathrm{L})$. India ink staining of the CSF was negative for cryptococcus meningitides, as was stool testing for liver flukes.

\section{Imaging studies}

Pulmonary computed tomography (CT) revealed small nodules, and hilar lymph node enlargement. MRI revealed countless, tiny, rounded, cystic lesions with high-signal intensity in a perivascular distribution on $\mathrm{T} 2$-weighted images, and low-signal intensity lesions on T1-weighted images in the bilateral cerebral cortex and subcortex, cerebellum, basal ganglia, cerebral peduncle, mammillary body, thalamus, and midbrain. T2 FLAIR sequences revealed that some of the lesions had homogeneous highsignal intensity, while other had low-signal intensity in the center and surrounding high-signal intensity. The diameter of the lesions ranged from $2.5-5 \mathrm{~mm}$. There was no visible edema surrounding any of the lesions. None of the lesions exhibited enhancement after the administration of intravenous contrast medium (Gd-DTPA). However, a focal nodule measuring about $8 \mathrm{~mm} \times 7 \mathrm{~mm}$ was seen in the hypophysis on $\mathrm{T} 2$-weighted images as high-signal intensity. This lesion exhibited uneven enhancement on T1-weighted images with contrast medium (Figure 1). Abdominal ultrasound showed no suspicious lesions in the solid organs. 

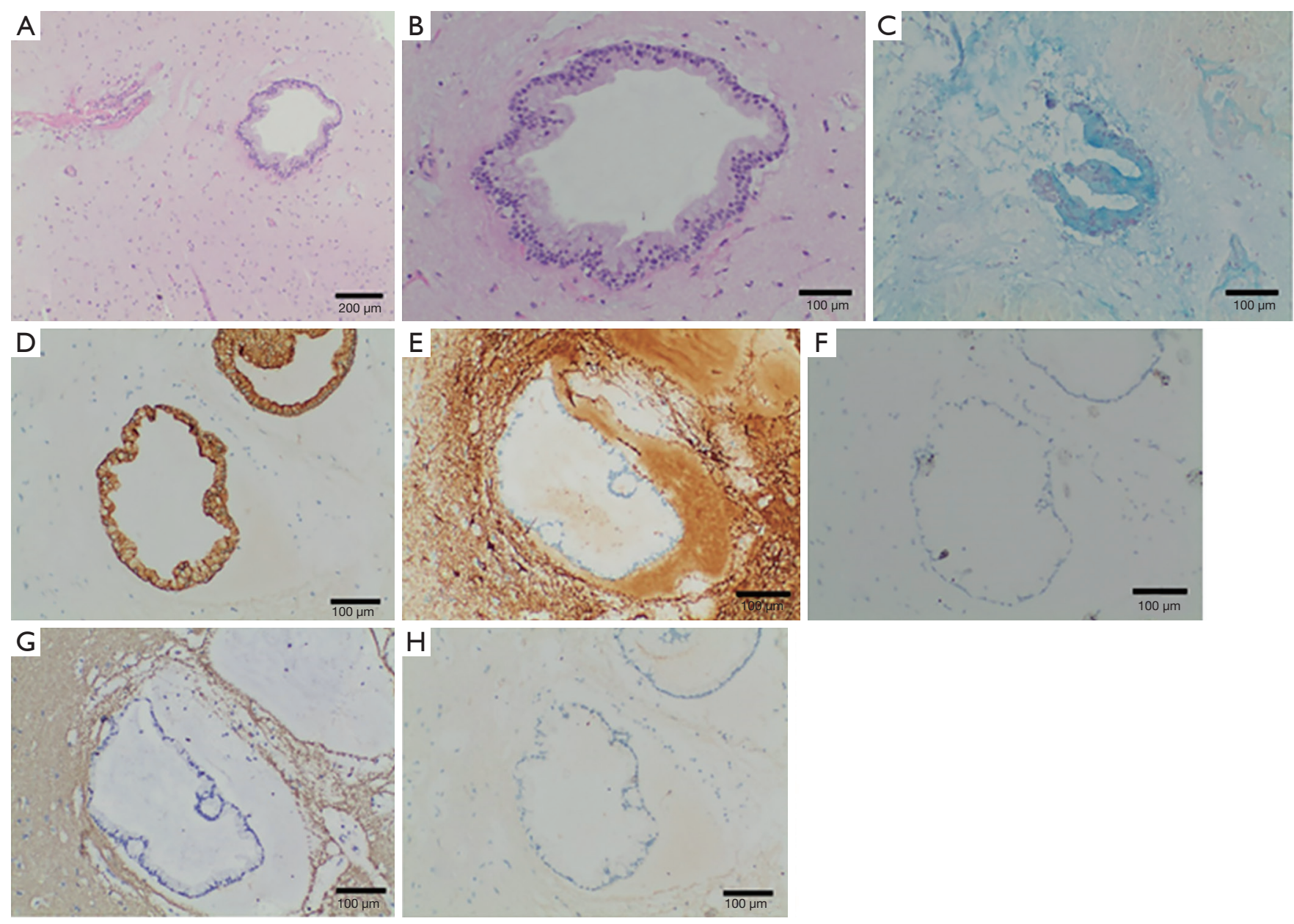

Figure 2 Histopathological examination after hematoxylin and eosin (HE) and immunohistochemical (IHC) staining. (A) HE $\times 40$. (B) HE $\times 100$. (C) AB mucus staining $\times 100$. (D) CK-7 ×100. (E) GFAP $\times 100$. (F) Ki-67 ×100. (G) Syn $\times 100$. (H) TTF- $\times 100$. HE staining showed well-differentiated glands were scattered in the brain tissue. At high magnification the glands were observed to be dilated, and mucus was observed in the cytoplasm of glandular epithelial cells. Cell atypia was not obvious. Other immunohistochemical staining was negative.

Based on the imaging findings, brain metastases and capillary telangiectasia were considered in the differential diagnosis.

\section{Brain biopsy}

To establish a definitive pathological diagnosis, a right frontal lobe biopsy was performed after obtaining the consent of the patient and his family. During the procedure under a surgical microscope, tissue of the cerebral cortex, sulcus, subcortical region appeared visually normal. However, the texture of the tissue that was resected for biopsy was fragile and prone to bleeding. The procedure was performed without any complications or bleeding, and no new neurological impairment in the patient was noted.

\section{Pathological findings}

Pathological examination of the biopsy specimen disclosed multiple metastatic nodules diffusely distributed in the brain tissue with a mucinous adenoid-liked pattern, which were consistent with well-differentiated adenocarcinoma. Immunohistochemistry (IHC) staining was performed with a Ventana Automated Immunostainer. The antibodies used included: Cytokeratin 7 (OV-TL12/30, 1:150, Neomarkers), thyroid transcription factor-1 (TTF-1) (8G7G3/1, 1:50, Neomarkers), Syn, GFAP, S-100, and CDX2. The neoplastic cells were positive for cytokeratin 7, and negative for TTF-1, 

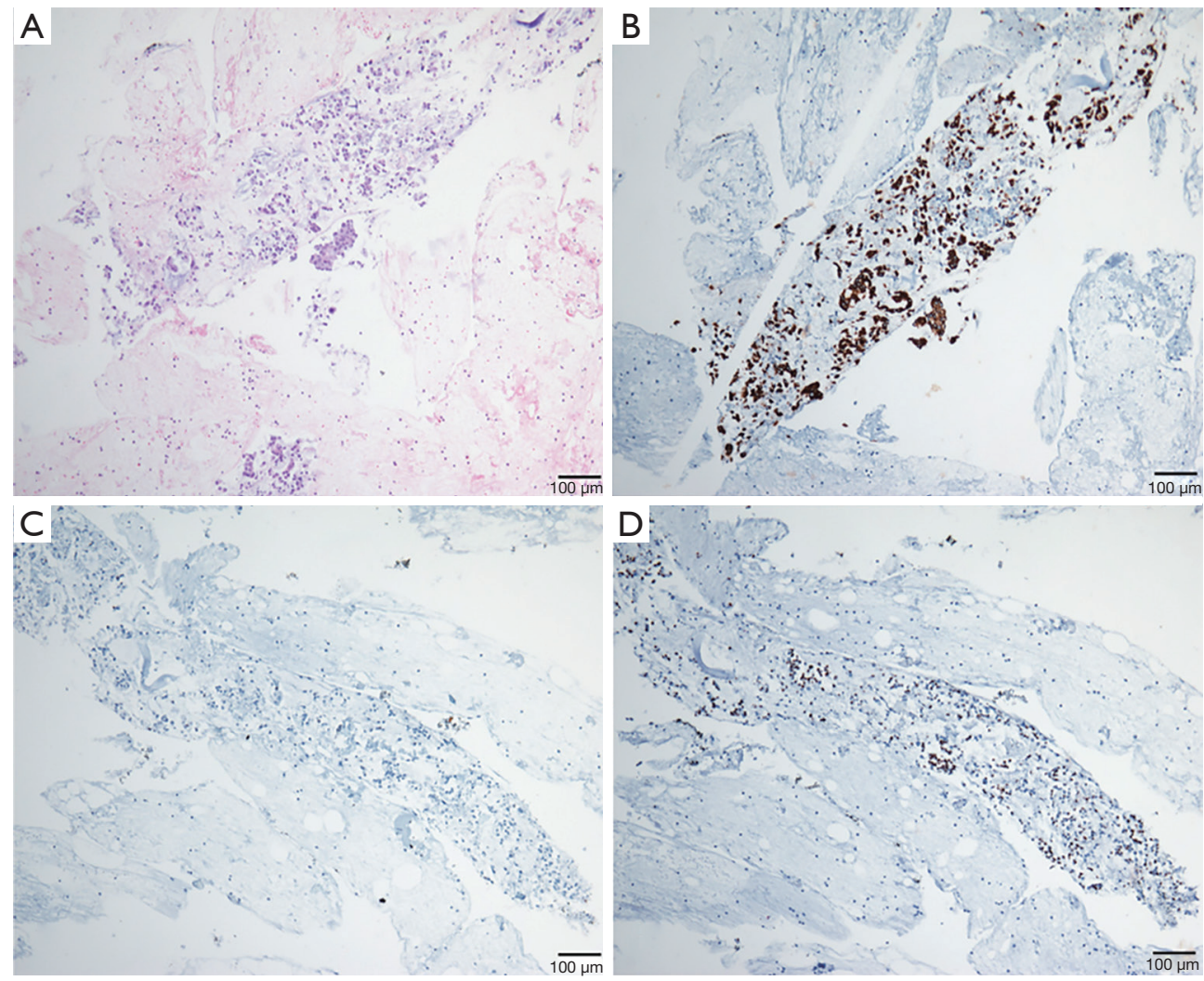

Figure 3 Histopathological examination of bronchi and bronchial lymph node biopsy performed with fiberoptic bronchoscopy. (A) HE $\times 100$. (B) CK-7 ×100. (C) Ki67 ×100. (D) TTF-1 ×100. HE staining showed that the glandular tissues exhibited abnormal differentiation. CK-7 and Ki67 staining showed no definitely positive results. TTF-1 staining showed darkly stained cells in the lesions, suggesting a positive result.

Syn, GFAP, S-100 and CDX2. These IHC results excluded miliary brain metastases from primary gastrointestinal adenocarcinoma, and were consistent with miliary brain metastasis from lung adenocarcinoma (Figure 2).

Review of the bronchial biopsy specimens and pathological results showed they were morphologically similar with the brain biopsy tissue, and the IHC staining results were the same except the bronchial tissue was positive for TTF-1 (Figure 3).

The patient and his family requested supportive treatments only, and he died 3 months after the diagnosis.

\section{Discussion}

Clinical manifestations of patients with miliary brain metastases can vary markedly, and a variety of common neurological deficits ranging from hemiparesis to unconsciousness may be present. In our review of the literature, 12,678 articles were identified using the search term "miliary metastatic lung (gland) cancer". However, careful review of the titles, abstract, and articles revealed there were only 6 that were similar to our case, i.e., miliary metastasis to the brain without lesion enhancement on MRI after administration of contrast (12-17). The details of the 6 cases are summarized in Table 1. Our patient presented with headache, dizziness, and vomiting, likely due to intracranial hypertension. While hydration helped to reduce the intracranial hypertension and moderate his symptoms, the response did not assist with the diagnosis. The absence of abnormalities in the CSF, and the atypical imaging findings, made distinguishing between an infective inflammatory process and tumor metastases difficult.

MRI is useful for the diagnosis of brain lesions. Typically, brain lesions enhance with the intravenous administration of contrast medium $(2-4,8-10,18,19)$. In most cases of miliary brain metastasis, the lesion are visible as high-intensity 


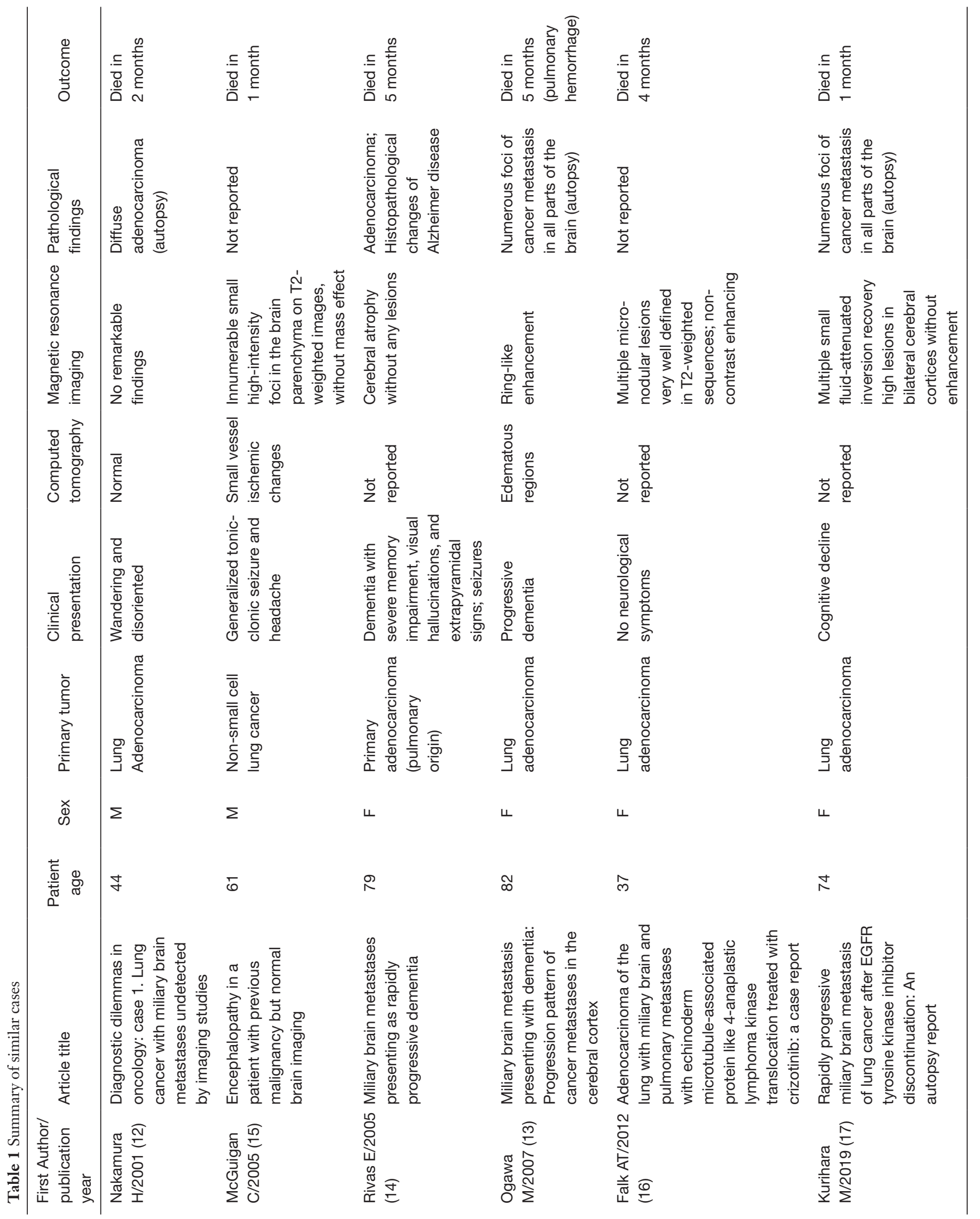


signals on T2-weighted images (2-7), and are enhanced on T1-weighted images after the administration of contrast medium(8-11). However, we found 6 cases in the literature where miliary brain metastases were not enhanced on MRI after the administration of contrast media (2-17).

In our patient, MRI revealed multiple high-signal intensity lesions on $\mathrm{T} 2$-weighted images, and low-signal intensity lesions on T1-weighted images in the cerebral hemispheres, cerebellum, and midbrain; however, the lesions did not enhance with the administration of contrast. Interestingly, a focal nodule was identified in the hypophysis on T2-weighted images as high-signal intensity, which exhibited uneven enhancement T1-weighted images after the administration of contrast. It has been speculated that contrast-enhanced MRI can fail to delineate metastatic lesions because the blood-brain barrier remains intact early in the disease process $(5,13)$ and the vascular system of the pituitary differs from that of the cerebral hemisphere. In addition, lesions can present as low-intensity signals on T1- weighted images and high-intensity signals on T2weighted images because of surrounding edema (10). In our patient, however, there was no mass effect and no edema was present surrounding any of the metastatic nodules.

Because of the atypical radiographic findings, we could not establish a definitive diagnosis and a brain biopsy was performed. Histopathological examination of the biopsy specimens confirmed the diagnosis of metastatic brain tumors. Importantly, the prior bronchial biopsy specimens were morphologically similar to the brain tissue specimens. Interestingly, the IHC staining results of the brain and bronchial tissue were the same except that the bronchial tissue was positive for TTF-1, whereas the brain tissue tested was negative.

TTF-1 is a transcription factor expressed in the thyroid and lung, and in some areas of the brain (20). TTF-1 is also found in thyroid and lung malignancies, and is useful as an IHC marker of tumors of thyroid and lung origin (20). TTF-1 expression or over-expression has been found to be an independent prognostic factor for non-small cell lung carcinoma $(21,22)$. In our case, the difference of TTF-1 expression between the bronchial tissue (positive expression) and the brain metastasis (negative expression) may suggest that the tumor may have undergone gene rearrangement during the metastatic process. This may explain why extensive miliary dissemination was present, and why the lesions were non-contrast enhancing.

The prognosis for patients with miliary brain metastasis is poor. Chemotherapy and whole-brain radiotherapy are generally ineffective $(3,5,13)$. Gefitinib has been reported to be a useful additive drug for resolution of the multiple metastatic lesions; however, further research on this treatment is necessary $(13,23,24)$.

\section{Conclusions}

Miliary brain metastasis is rare, and the presentation is variable. Diagnosis can be complicated when imaging findings are atypical, i.e., lesions on MRI do not enhance with the administration of contrast medium. In these cases, a biopsy of brain tissue may be necessary for a definitive diagnosis. IHC staining for TTF-1 may also aid in the diagnosis. The prognosis for patients with miliary brain metastasis is poor.

\section{Acknowledgments}

Funding: None.

\section{Footnote}

Reporting Checklist: The authors have completed the CARE reporting checklist. Available at http://dx.doi.org/10.21037/ tcr-20-1898

Conflicts of Interest: All authors have completed the ICMJE uniform disclosure form (available at http://dx.doi. org/10.21037/tcr-20-1898). The authors have no conflicts of interest to declare.

Ethical Statement: The authors are accountable for all aspects of the work in ensuring that questions related to the accuracy or integrity of any part of the work are appropriately investigated and resolved. This study was approved by the institutional review board (IRB) of our hospital \{approval no. [2020]-02-164-01\}. All procedures performed in studies involving human participants were in accordance with the ethical standards of the institutional and/or national research committee(s) and with the Helsinki Declaration (as revised in 2013). Written informed consent was obtained from the patient.

Open Access Statement: This is an Open Access article distributed in accordance with the Creative Commons Attribution-NonCommercial-NoDerivs 4.0 International License (CC BY-NC-ND 4.0), which permits the noncommercial replication and distribution of the article with 
the strict proviso that no changes or edits are made and the original work is properly cited (including links to both the formal publication through the relevant DOI and the license). See: https://creativecommons.org/licenses/by-nc-nd/4.0/.

\section{References}

1. Madow L, Alpers BJ. Encephalitic form of metastatic carcinoma. AMA Arch Neurol Psychiatry 1951;65:161-73.

2. Floeter MK, So YT, Ross DA, et al. Miliary metastasis to the brain: Clinical and radiologic features. Neurology 1987;37:1817-8.

3. Bhushan C. "Miliary" metastatic tumors in the brain. Case report. J Neurosurg 1997;86:564-6.

4. Nishimura H, Nishimura T, Tachibana H SM. A case with miliary metastatic brain tumor. Shinkeinaika 1997;53:173-4.

5. Nemzek W, Poirier V, Salarnat MS, et al. Carcinomatous encephalitis (Miliary metastases): Lack of contrast enhancement. Am J Neuroradiol 1993;14:540-2.

6. Ruppert AM, Stankoff B, Lavolé A, et al. Miliary brain metastases in lung cancer. J Clin Oncol 2010;28:e714-6.

7. Olsen WL, Winkler ML, Ross DA. Carcinomatous encephalitis: CT and MR findings. Am J Neuroradiol 1987;8:553-4.

8. Iguchi Y, Mano K, Goto Y, et al. Miliary brain metastases from adenocarcinoma of the lung: MR imaging findings with clinical and post-mortem histopathologic correlation. Neuroradiology 2007;49:35-9.

9. Shirai H, Imai S, Kajihara Y, et al. MRI in carcinomatous encephalitis. Neuroradiology 1997;39:437-40.

10. Kahveci R, Gürer B, Kaygusuz G, et al. Miliary brain metastases from occult lung adenocarcinoma: Radiologic and histopathologic confirmation. J Neurosci Rural Pract 2012;3:386-9.

11. Dziadziuszko K, Szurowska E, Pienkowska J, et al. Miliary brain metastases in a patient with ROS1-rearranged lung adenocarcinoma: A case report. J Thorac Oncol 2014;9:e34-6.

12. Nakamura H, Toyama M, Uezu K, et al. Diagnostic dilemmas in oncology: Case 1. Lung cancer with miliary brain metastases undetected by imaging studies. J Clin Oncol 2001;19:4340-1.

13. Ogawa M, Kurahashi K, Ebina A, et al. Miliary brain metastasis presenting with dementia: Progression pattern of cancer metastases in the cerebral cortex. Neuropathology 2007;27:390-5.
14. Rivas E, Sanchez-Herrero J, Alonso M, et al. Miliary brain metastases presenting as rapidly progressive dementia. Neuropathology 2005;25:153-8.

15. McGuigan C, Bigham S, Johnston D, et al. Encephalopathy in a patient with previous malignancy but normal brain imaging. Neurology 2005;65:165.

16. Falk AT, Poudenx M, Otto J, et al. Adenocarcinoma of the lung with miliary brain and pulmonary metastases with echinoderm microtubule-associated protein like 4-anaplastic lymphoma kinase translocation treated with crizotinib: A case report. Lung Cancer 2012;78:282-4.

17. Kurihara M, Koda H, Aono H, et al. Rapidly progressive miliary brain metastasis of lung cancer after EGFR tyrosine kinase inhibitor discontinuation: An autopsy report. Neuropathology 2019;39:147-55.

18. Ribeiro HB, De Paiva TF, Mamprin GPR, et al. Carcinomatous encephalitis as clinical presentation of occult lung adenocarcinoma: Case report. Arq Neuropsiquiatr 2007;65:841-4.

19. Sawamura Y, Murata J TK. Miliary brain metastases. Shinkeinaika 2000;53:2-3.

20. Phelps CA, Lai SC, Mu D. Roles of Thyroid Transcription Factor 1 in Lung Cancer Biology. Vitam Horm 2018;106:517-44.

21. Takeuchi A, Oguri T, Yamashita Y, et al. TTF-1 Expression predicts the merit of additional antiangiogenic treatment in non-squamous non-small cell lung cancer. Anticancer Res 2018;38:5489-95.

22. Kim JH, Kim HS, Kim BJ, et al. Prognostic impact of TTF-1 expression in non-squamous non-small-cell lung cancer: a meta-analysis. J Cancer 2018;9:4279-86.

23. Cappuzzo F, Ardizzoni A, Soto-Parra H, et al. Epidermal growth factor receptor targeted therapy by ZD 1839 (Iressa) in patients with brain metastases from non-small cell lung cancer (NSCLC). Lung Cancer 2003;41:227-31.

24. Ceresoli GL, Cappuzzo F, Gregorc V, et al. Gefitinib in patients with brain metastases from non-small-cell lung cancer: A prospective trial. Ann Oncol 2004;15:1042-7.

Cite this article as: Huang Z, Xie D, Yang P, Song E, Zhang J, Chen J, Qin F. Miliary brain metastases from lung adenocarcinoma as non-enhancing lesions on MRI: a case report and literature review. Transl Cancer Res 2021;10(2):11621168. doi: $10.21037 /$ tcr-20-1898 